\title{
New Species of Extinct Rails (Aves: Rallidae) from Archaeological Sites in the Marquesas Islands, French Polynesia ${ }^{1}$
}

\author{
Feremy 7. Kirchman ${ }^{2,4}$ and David W. Steadman ${ }^{3}$
}

\begin{abstract}
We examined 53 bones of rails (Rallidae), previously referred to Gallirallus n. spp., from archaeological sites on four islands in the Marquesas Islands, French Polynesia. We describe three new, extinct, flightless species of Gallirallus: G. roletti (Tahuata), G. gracilitibia (Ua Huka), and G. epulare (Nuku Hiva). Two bones from Hiva $\mathrm{Oa}$, although probably representing another extinct species of Gallirallus, are regarded as an inadequate basis for describing a species. At first human contact, the genus Gallirallus probably included many scores if not hundreds of flightless species on islands from the far western $\mathrm{Pa}$ cific (Okinawa, Philippines, Halmahera) eastward across most of Oceania. As currently understood, the Marquesas Islands represent the eastern range limit of Gallirallus.
\end{abstract}

Bird bones from Pacific islands (Figure 1) have revealed extensive Late Holocene extinctions of land birds following prehistoric human arrival (James and Olson 1991, Olson and James 1991, Steadman 1995, 2006, Worthy and Holdaway 2002). Radiocarbon chronologies of stratified zooarchaeological assemblages indicate that, in the earliest stages of occupation, people consumed a relatively large number of indigenous birds (Steadman and Rolett 1996, Steadman et al. 2002). In East Polynesia, from 50 to $100 \%$ of the species of land birds were extirpated from most islands. Especially prevalent among the extinct species are rails (Order Gruiformes, Family Rallidae), most of which were flightless species endemic to single is-

\footnotetext{
${ }^{1}$ Financial support came from a McGlaughlin Fellowship to J.J.K. from the University of Florida College of Liberal Arts and Sciences, and NSF grants EAR9714819 and DEB-0228682 to D.W.S. Manuscript accepted 14 February 2006.

${ }^{2}$ New York State Museum, 3140 CEC, Albany, NY 12230.

${ }^{3}$ Florida Museum of Natural History, University of Florida, Gainesville, Florida 32611.

${ }^{4}$ Corresponding author (phone: 518-474-1441; e-mail: jkirchman@mail.nysed.gov).
}

Pacific Science (2007), vol. 61, no. 1:145-163

(C) 2007 by University of Hawai'i Press

All rights reserved lands. These ground-nesting birds were especially vulnerable to predation by people and their introduced rats, pigs, and dogs.

Nearly all of the known extinct $(\dagger)$ species of flightless rails have been placed in widespread, extant genera, including $7-10$ species of Porzana from the Hawaiian Islands alone (Olson and James 1991); one to several species each of Gallirallus, Gallinula, Porphyrio, and Fulica from New Zealand (Worthy and Holdaway 2002); and many species of Porzana, Gallirallus, and Porphyrio from the rest of Oceania (Steadman 1995, 2006, Kirchman and Steadman 2005, 2006). Thus flightlessness, which became terminally maladaptive only after Polynesians and their commensals colonized the Pacific, evolved independently in insular rails numerous times, even within archipelagos. Of the crudely estimated hundreds (Pimm et al. 1994, Livezey 2003) to 500 to 1,600 (Steadman 2006) to nearly 2,000 (Steadman 1995) species of flightless rails that once existed across the Pacific, fewer than 20 have been described (Worthy 2004). Here we describe the prehistoric bones of rails from archaeological sites on four islands in the Marquesas Islands.

\section{Marquesan Archaeological Sites}

Centered at ca. $9^{\circ} \mathrm{S}$ and $140^{\circ} \mathrm{W}$, the Marquesas Islands lie ca. $500 \mathrm{~km}$ northwest of the Tuamotu Archipelago and 1,400 km north- 


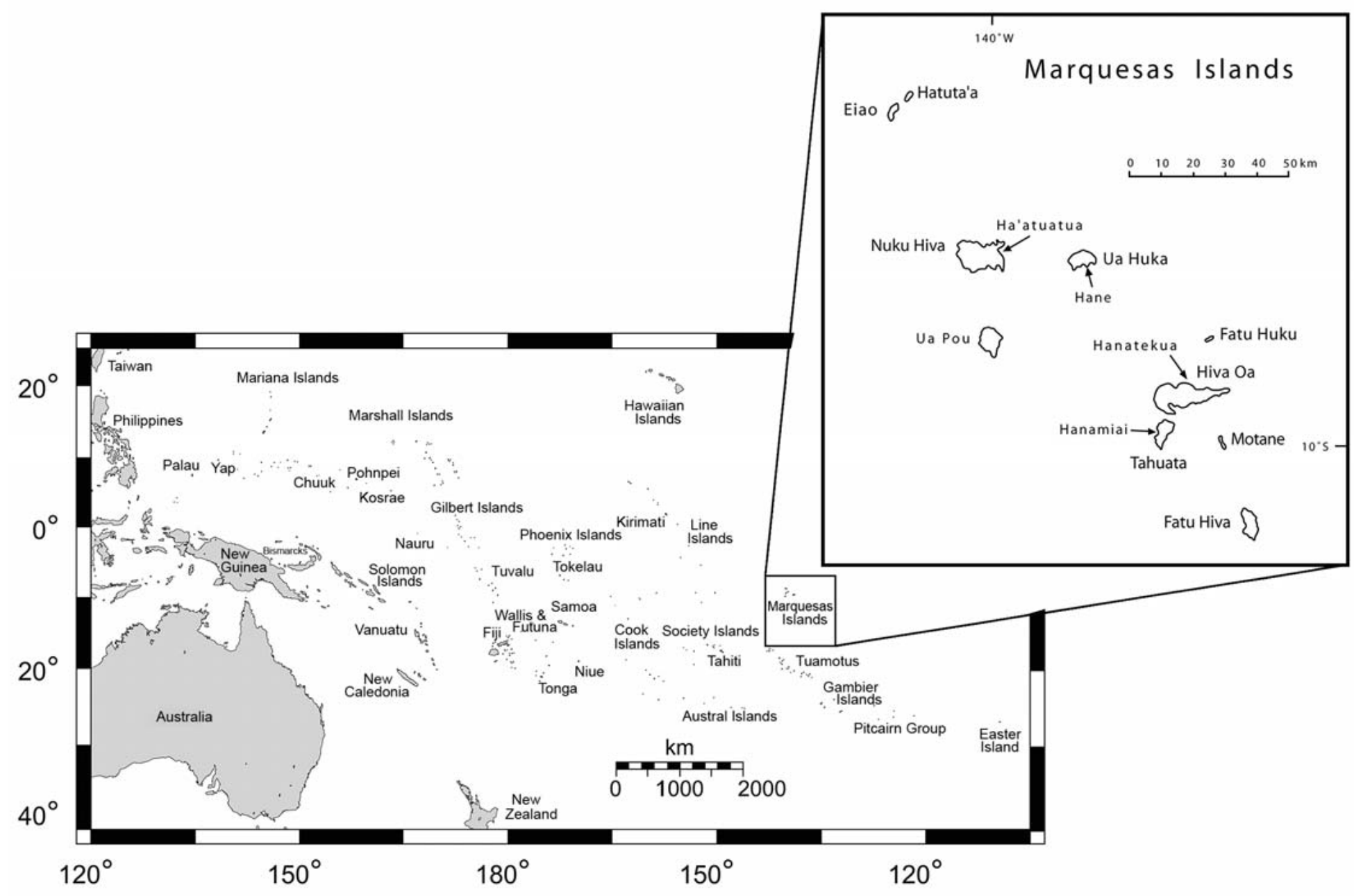

FIGURE 1. Oceania, showing location of the Marquesas Islands and location of the four archaeological sites discussed in this paper. 
east of the Society Islands, which are the nearest high islands (Figure 1). The Marquesas consist of 10 major eroded volcanic islands with surface areas that range from $<2$ to $330 \mathrm{~km}^{2}$ and maximum elevations from 420 to $1,252 \mathrm{~m}$. Minimum interisland distances among these 10 islands range from 3 to $21 \mathrm{~km}$. Hiva $\mathrm{Oa}$ and nearby Tahuata probably were connected to each other during the last Pleistocene glacial interval, whereas Nuku Hiva and Ua Huka remained as separate islands even when the sea level was 120 $130 \mathrm{~m}$ lower than at present.

The archaeological specimens reported here are 53 bones that were previously referred to "Gallirallus new spp." or "Gallirallus undescribed spp." by Steadman and Rolett (1996) and Steadman $(1989,2006)$. The sites represent early human occupations developed in coastal calcareous sands on four islands. The Hanamiai site on Tahuata was excavated by B. V. Rolett in 1984-1985 (Rolett 1998). The sediment excavated at Hanamiai was screen-washed through 1/8-inch $(3.2-\mathrm{mm})$ mesh, producing 716 identifiable bird bones, among which are 70 land bird bones from 10 species (Steadman and Rolett 1996). The Hanatekua Rockshelter on Hiva Oa was excavated in 1967-1968 by Y. H. Sinoto and P. Bellwood (Bellwood 1972), yielding 146 land bird bones from seven species (Steadman 2006). The Hane Dune site (often called merely the Hane site) on Ua Huka, excavated by Y. H. Sinoto in the 1960s (Sinoto 1966, 1970, 1979), is the richest Marquesan site in terms of faunal remains, having yielded more than 12,000 identifiable bird bones, including 2,187 land bird bones from 17 species (Steadman 2006). The Ha'atuatua Dune site (often called merely the Ha'atuatua site) on Nuku Hiva was excavated initially by R. Suggs in 1956 and 1958 (Suggs 1961) and in 19921994 by B. V. Rolett and E. Conte (Rolett 1998). The combined excavations at Ha'atuatua Dune yielded 27 bones of land birds belonging to nine species (Steadman 2006).

Based on radiocarbon $\left({ }^{14} \mathrm{C}\right)$ dates from reliable stratigraphic levels, the initial human occupation for Tahuata, Hiva Oa, and Nuku Hiva was ca. 1,000 yr B.P. (years before present) and ca. 1,350 yr B.P. for Ua Huka (Ro- lett 1998). Hane Dune (Ua Huka) is the earliest well-dated site in the Marquesas. Estimates that people arrived in the Marquesas as early as 2,000 yr B.P. (e.g., Sinoto 1979) are from single ${ }^{14} \mathrm{C}$ samples from poorly established stratigraphic contexts or are based on ${ }^{14} \mathrm{C}$ dates with suspected laboratory errors (Rolett 1998).

\section{MATERIALS AND METHODS}

Skeletons used for comparison with fossils are from the American Museum of Natural History (АмNH); Bernice P. Bishop Museum (врвм); Florida Museum of Natural History, University of Florida (Uf); National Museum of Natural History, Smithsonian Institution (USNM); National Museum of New Zealand (NMnz); Départment de Archéologie, Centre Polynésien des Sciences Humaines, Tahiti (DAPT); University of Michigan Museum of Zoology (ummz); Thomas Burke Memorial Museum, University of Washington (Uwвм); and Yale Peabody Museum (YPM). We examined these modern specimens: Porzana tabuensis, ишвм 42501, 42528; Rallus longirostris, UF 40956, 24200; Gallirallus striatus, USNM 85892, 343214, 559919, урм 107205; G. torquatus, uMMz 228275, 228279, 228280, AMNH 17715-17717, USNM 290445; G. owstoni, UF 39918-39921, 39256, 42968, umMz 215472, USNM 561968, 611816, 612616, 613738614744, 614233-614235, 614771, 614772; G. australis, UF 24326, 24327, YРM 102249, 110760, 110789, 110790, 110844; G. philippensis, uf 39854, 39855, 42902, 42935, 42934, 42933, 43224, ишвм 42865, 42866, 42863, USNM 560651, 560791, 620196; G. ["Nesoclopeus"] woodfordi, UF 39399, 39406, 39409, 39547, 39556, 39574; Amaurornis olivaceus, UF 40216; A. phoenicurus, uf 24387; Porphyrio porphyrio, UF 39388,39407 ; $P$. martinicus, UF 39927, 42418, 42419; Gallinula chloropus, UF 39927. We also examined these fossil specimens: †Gallirallus buiatua tarsometatarsus, NMNZ S37708 (holotype), ulna, NMnz S37709 (paratype), femur, NMNZ S37710 (paratype), tibiotarsus, NMNZ S37711 (paratype); †G. ripleyi rostrum, uf 55596, coracoid, UF 54711, USNM 402896 (paratype), humeri, uf 51402, 55752, ulnae, UF 55215, 54901, femur, UF 
51320, tibiotarsi, UF 55283, 59574, USNM 402895 (holotype), tarsometatarsi, uf 55223 , USNM 402895 (holotype); †G. storrsolsoni rostra, вРвм 166036 (holotype), DAPт 21, humerus, врвм 166022, ulnae, вРвм 166033, femur, DAPT 27/105, tibiotarsi, вРвм 166023 , DAPT 119 , tarsometatarsi, вРВм 166034, DАРТ $7 ; \dagger G$. vekamatolu rostrum, uf 52292 , mandibles, uf 51836, 52525, coracoids, uf 52204, 52966, scapulae, uf 52179, 52318, humeri, UF 52333, 52707, ulna, uf 51734, femora, UF 52058, 52518, tibiotarsi, uf 51729, 52211, tarsometatarsi, uF 52002, 52137; †Porphyrio paepae femora, врвм 165649, 166434, tibiotarsus, врвм 165651; and $\dagger P$. mcnabi femora, врвм 166031 (holotype), DAPT 39, DAPT 53 (paratypes).

Measurements were taken with electronic digital calipers and rounded to the nearest $0.1 \mathrm{~mm}$. Osteological terminology follows Baumel and Witmer (1993).

\section{COMPARATIVE OSTEOLOGY AND SYSTEMATICS}

\section{Family Rallidae}

The phylogeny of genera in the Rallidae is not well resolved. As a starting point for genus-level classification, we regard all "typical long-billed rails" (as opposed to swamphens, moorhens, coots, and crakes) from Oceania as species of Gallirallus sensu lato. We agree with Worthy (2004) that the recently discovered fossil rail $\dagger$ Vitirallus watling $i$ from Fiji belongs in a separate genus despite having a long (though decurved) bill and sharing other osteological similarities with Gallirallus. Our classification agrees with that of Olson (1973) by including in Gallirallus the species australis, philippensis, owstoni, okinawae, †wakensis, torquatus, insignis, sylvestris, $\dagger$ dieffenbachii, †modestus, striatus, and pectoralis. Among these species, only G. philippensis, $G$. torquatus, G. striatus, and G. pectoralis are volant. Olson (1973) provisionally retained $G$. woodfordi and $\dagger G$. poecilopterus in Nesoclopeus but highlighted their close affinity with Gallirallus, within which we classify these two species. This treatment differs from that of Livezey $(1998,2003)$, who acknowledged the difficulty of establishing relationships in this group solely on the basis of osteology but who divided the 14 species mentioned here among Gallirallus, Nesoclopeus, Tricholimnas, Cabalus, and Habropteryx.

As defined herein, Gallirallus includes at least 17 named flightless species endemic to single islands or islands connected during periods of lowered sea level. This total includes eight extant species (G. australis of New Zealand; G. sylvestris of Lord Howe Island; $G$. owstoni of Guam; G. okinawae of Okinawa; G. insignis of New Britain; G. woodfordi s.l. of the Solomon Islands [minimally Bougainville, Santa Isabel, and Guadalcanal]; G. rovianae of New Georgia, Solomon Islands; and G. calayanensis of Calayan, Philippines), five historically extinct species $(\dagger G$. wakensis of Wake Island; $\dagger G$. lafresnayanus of New Caledonia; $\dagger$ G. modestus of the Chatham Islands, New Zealand; †G. poecilopterus of Viti Levu, Fiji; and $\dagger G$. pacificus of Tahiti, Society Islands), and four previously named prehistoric species ( $\dagger$. buiatua from noncultural Holocene cave deposits on Niue [Steadman et al. 2000]; $† G$. ripleyi from cultural and noncultural late Holocene sites on Mangaia, Cook Islands [Steadman 1987]; $\dagger G$. vekamatolu from precultural strata on 'Eua, Kingdom of Tonga [Kirchman and Steadman 2005]; and $\dagger G$. storrsolsoni from cultural deposits on Huahine, Society Islands [Kirchman and Steadman 2006]).

\section{Genus Gallirallus Lafresnaye, 1841}

We refer the 53 fossils from the Marquesas Islands to Gallirallus rather than to other genera of oceanic rails on the basis of the following characters. Rostrum: long, narrow, and shallow with elongate nares. Mandible: cotyla lateralis narrow and concave, fossa for condylus medialis quadratum (the main articulation surface in the os articulare) shallow and wide. Coracoid: acrocoracoid extends medially over the sulcus musculo supracoracoidei such that the foramen triosseum is less open cranially. Scapula: facies articularis clavicularis relatively small and oriented at a more obtuse angle from corpus scapulae. Humerus: fossa pneumotricipitalis deep and wide with prominent crus ventrale fossae. Ulna: thin in 
cranial aspect with rectangular (rather than rounded) margo cranialis. Pelvis: ala preacetabularis ilii broadly continuous with crista dorsalis of synsacrum. Femur: distal end of corpus femoris becomes gradually wider; condylus medialis subcircular in medial aspect; impresso ansae musculo iliofibularis abuts suclus fibularis; rotolar groove broad. Tibiotarsus: craniolateral and craniomedial margins of corpus tibiotarsi rounded rather than sharp; impresso ligamentum collateralis medialis deep and wide; facies articularis femoris large; depressio epicondylaris lateralis deep; condylus medialis subcircular in medial aspect. Tarsometatarsus: corpus tarsometatarsi much wider than deep; medial sulcus hypotarsi not enclosed; fossa parahypotarsalis medialis shallow in proximal aspect; fossa metatarsi I short and shallow; crista plantaris mediana slopes gradually (not steeply) to hypotarsus; distal end of trochlea metatarsi tertii sloped toward medial trochlea; cotyla medialis is rectangular in proximal aspect with flat (not rounded) dorsal margin.

$\dagger$ Gallirallus roletti Kirchman \& Steadman, n. sp.

Figures $2 A, 3 A, 4 A, 5 A, 6 A$

holotype. Associated complete right femur, врвм 166447; right tibiotarsus, вРвм 166446; right tarsometatarsus, вРвм 166448; and pedal phalages, врвм 166449,166450 (Figure 2A). From the Hanamiai archaeological site, Tahuata, Marquesas Islands. Collected by B. V. Rolett and colleagues in 1984-1985.

paratypes. Distal rostrum, врвм 166456 (Figure $3 A$ ); left articular, врвм 166458; right articular, вРвм 166542; distal dentaries, вРвм 166436, 166439, 166457; left coracoid (humeral end), врвм 166455; left proximal femur, врвм 166435; right femur shaft, вРвм 166445; left distal tibiotarsus, врвм 166444; left tibiotarsi shafts, врвм 166437, 166452; right distal tibiotarsus, врвм 166438; left distal tarsometatarsus, врвм 166451; nearly complete right tarsometatarsus, в ввм 166440; right proximal tarsometatarsus, вРвм 166454; right tarsometatarus shaft, вРвм 166441. All are from the same locality as the holotype.
Diagnosis. A medium-sized, flightless species of Gallirallus (Table 1) distinguished from all examined congeners (except where similarities are noted) as follows. Rostrum (Figure 3A): more robust and deep with height to width ratio (at anterior margin of nares) of 1.24 ( $\leq 1.03$ in all other species of Gallirallus); in ventral aspect, trough in os premaxilare deep and wide, crista tomialis thin and sharp. Mandible: in caudal aspect, fossa caudalis with straight lateral and medioventral sides and a deep, narrow groove on top (ventral) side; pars symphysialis long, with rami sloped steeply to form a V-shaped (rather than U-shaped) trough in cranial aspect. Femur (Figures 2A, 4A): mediodistal margin of neck deeply excavated below facies articularis acetabularis, forming a sulcus in anterior aspect; in ventral aspect, trochanter femoris forms a prominent right angle with facies articularis antitrochanterica; the most proximal impressiones obturatoriae deep and long, forming a groove parallel to the crista trochanteris; in medial aspect, corpus femorus stout and straight, especially on leading edge (facies cranialis). Tibiotarsus (Figures $2 A$, $5 A$ ): fossa retropatellaris narrow but deep; impresso ligamentum collateralis medialis shallow; fossa flexoria shallow; crista fibularis short but broad, projecting farther from corpus tibiotarsis at distal end than in all except G. owstoni; tuberculum retinaculi musculo fibularis prominent (as in $\dagger G$. storrsolsoni and G. torquatus) but not forming a tube (as in $G$. woodfordi); condylus medialis and condylus lateralis large relative to width and depth of corpus tibiotarsus. Tarsometatarsus (Figures $2 A, 6 A$ ): proportionately stout, although not so much as in $\dagger G$. vekamatolu or $\dagger G$. ripleyi; sulcus extensorius deeply concave; corpus tarsometatarsi shallow relative to its breadth; facies medialis thin in medial aspect, especially proximally (approaching the condition in Amaurornis olivaceus or Porphyrio spp.); sulcus proximal to the foramen vasculare distale short and shallow; distal trochleae large and widely splayed.

ETYMOLOGY. Named in honor of Barry V. Rolett, whose outstanding research in the Marquesas Islands has been of great importance to both biologists and archaeologists. 


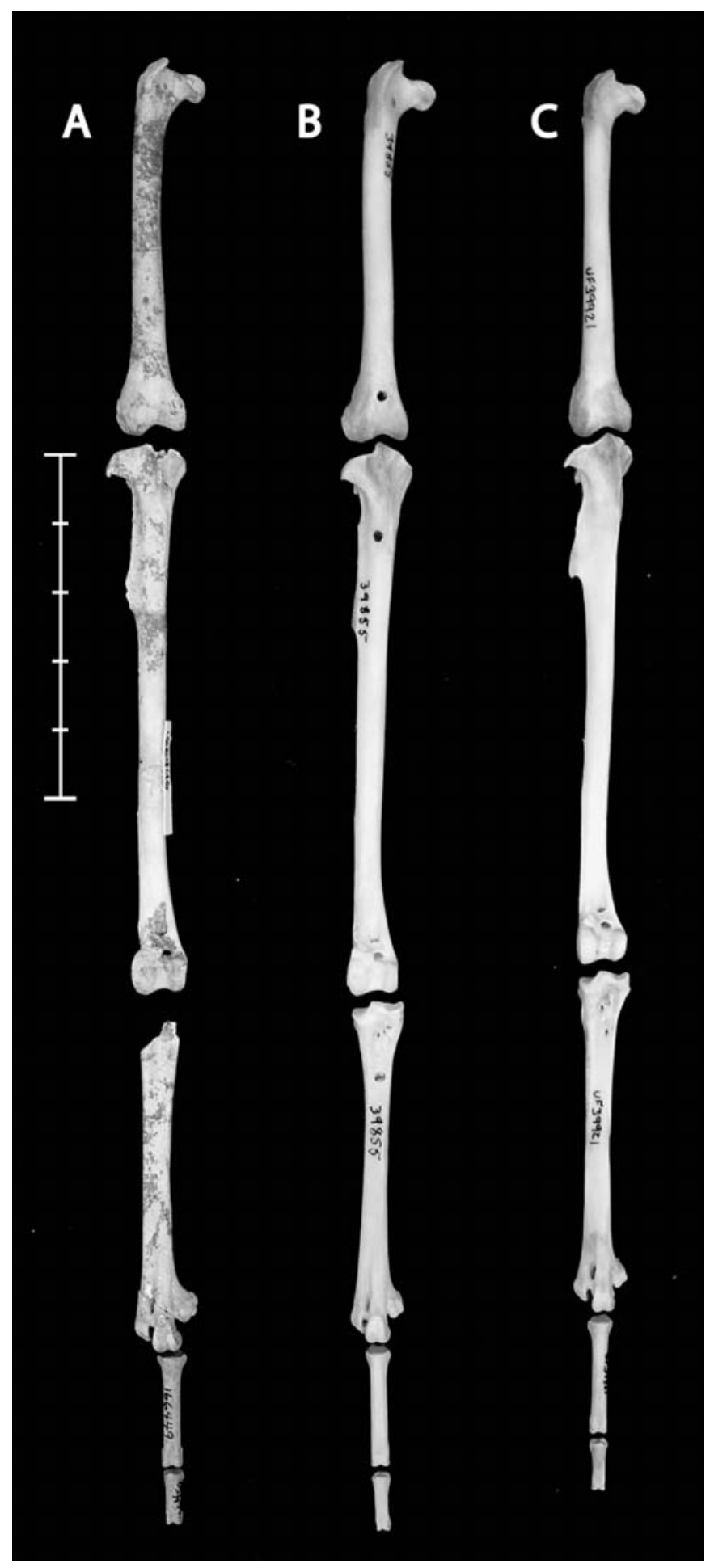

FIGURE 2. $A$, †Gallirallus roletti holotype consisting of associated femur (врвм 166447), tibiotarsus (врвм 166446), tarsometatarsus (врвм 166448), and two pedal phalanges (врвм 166449, 166450), top to bottom, respectively, Tahuata, Marquesas Islands. Shown with the same elements from B, G. philippensis (uf 39855, Tutuila, Samoa), and C, G. owsstoni (uf 39921, Guam, Mariana Islands). Scale bar $=5 \mathrm{~cm}$. 


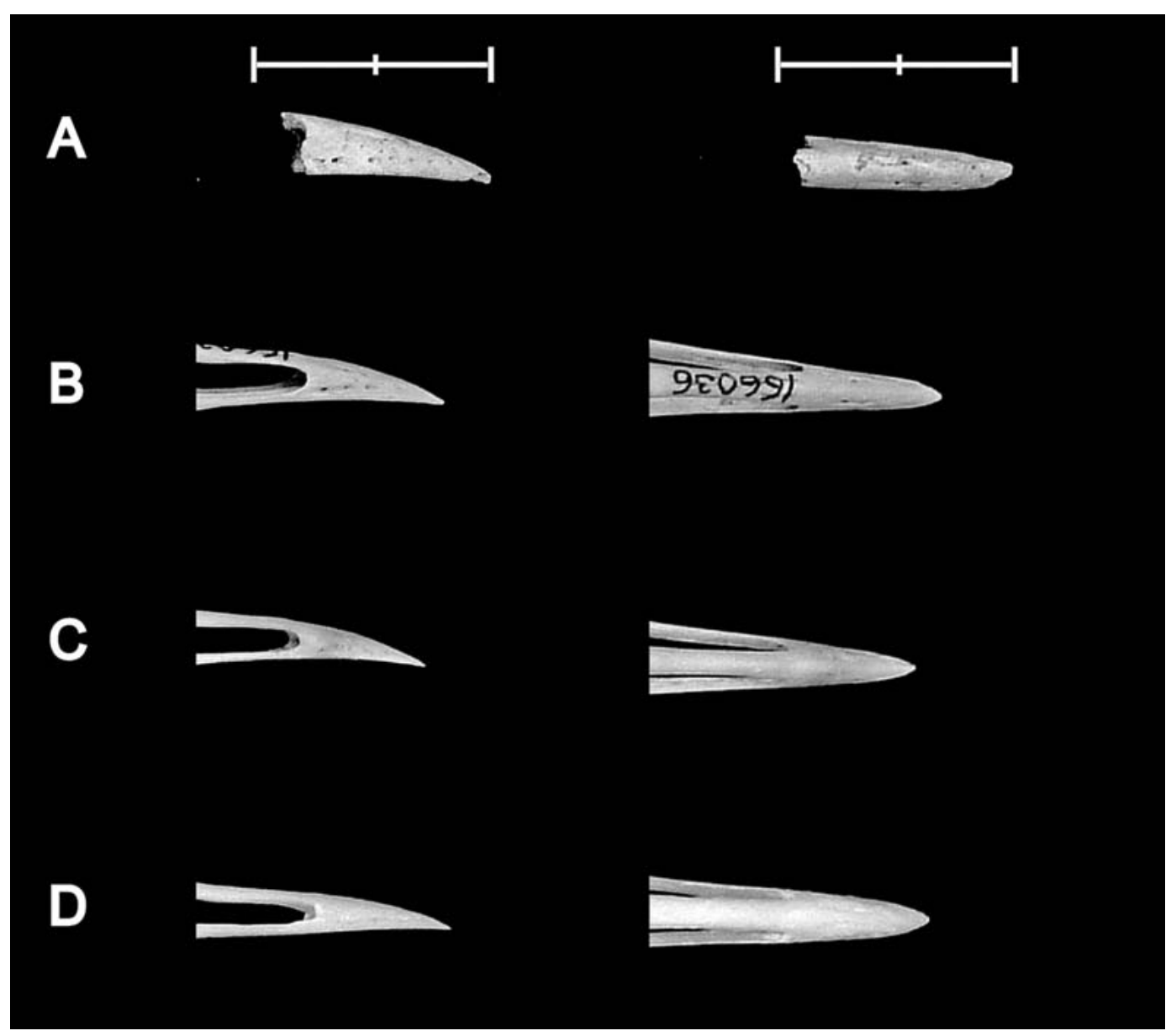

Figure 3. Rostra of $A, \dagger$ Gallirallus roletti (врвм 166456, Tahuata, Marquesas Islands); $B, \dagger G$. storrsolsoni (holotype, врвм 166036, Huahine, Society Islands); C, G. philippensis (uf 39855, Tutuila, Samoa); and D, G. owstoni (uf 39921, Guam, Mariana Islands) in lateral (left) and dorsal (right) aspects. Scale bars $=2 \mathrm{~cm}$.

In particular, his careful excavations at the Hanamiai site yielded the most extensive and useful series of Gallirallus bones from the Marquesas Islands.

REMARKs. In overall size, †Gallirallus roletti resembles G. owstoni, G. philippensis, G. striatus, and G. torquatus. It is larger than $\dagger G$. ripleyi and $\dagger G$. wakensis, and smaller than G. australis, $\dagger$ G. vekamatolu, and G. woodfordi. The material from Hanamiai represents four individuals, minimally. Lacking the sternum, scapula, and elements of the forelimb, our only evidence that $\dagger G$. roletti was flightless comes from the relatively small size of the coracoid (врвм 166455) and the larger, more open shape and more medial position of the cotyla scapularis on the dorsal surface of the coracoid, which more closely resembles the condition in flightless rather than volant species of Gallirallus. This specimen is pitted over much of its surface, indicating that it may represent a juvenile bird. Comparing this coracoid among those of known juveniles and adults in G. philippensis (volant) and G. owstoni (flightless) suggests that врвм 166455 is from a bird 3 to 4 months old and that the cora- 


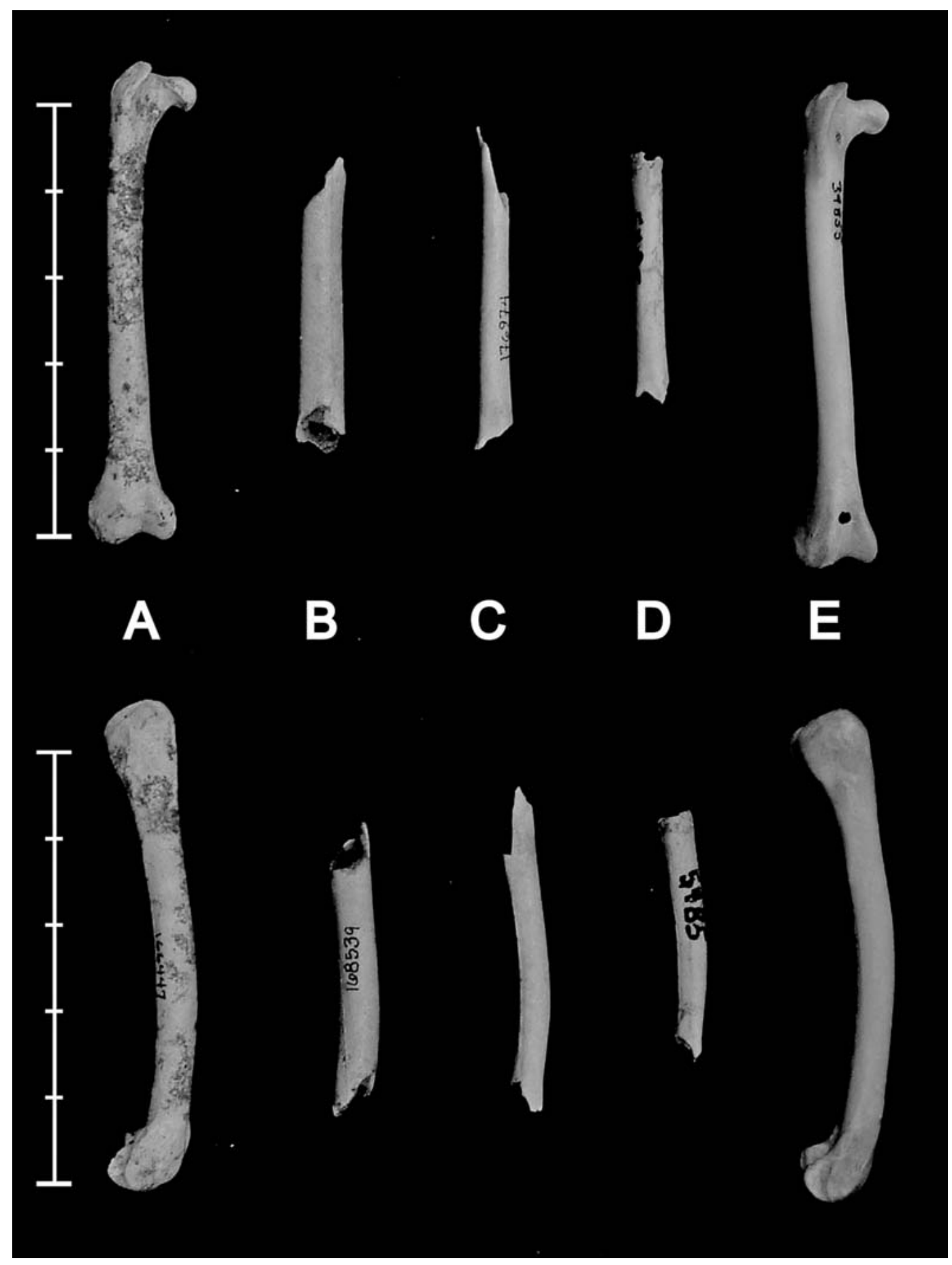

FIGURE 4. Femora of $A, \dagger$ Gallirallus roletti (holotype, врвм 166447, Tahuata, Marquesas Islands); $B$, cf. $\dagger$ Gallirallus sp. (врвм 168539, Hiva Oa, Marquesas Islands); $C, \dagger G$. gracilitibia (врвм 176974, Ua Huka, Marquesas Islands); $D, \dagger \mathrm{G}$. epulare (врвм 181659, Nuku Hiva, Marquesas Islands); and E, G. philippensis (uf 39855, Tutuila, Samoa) in dorsal (top) and ventral (bottom) aspects. Scale bars $=5 \mathrm{~cm}$. 


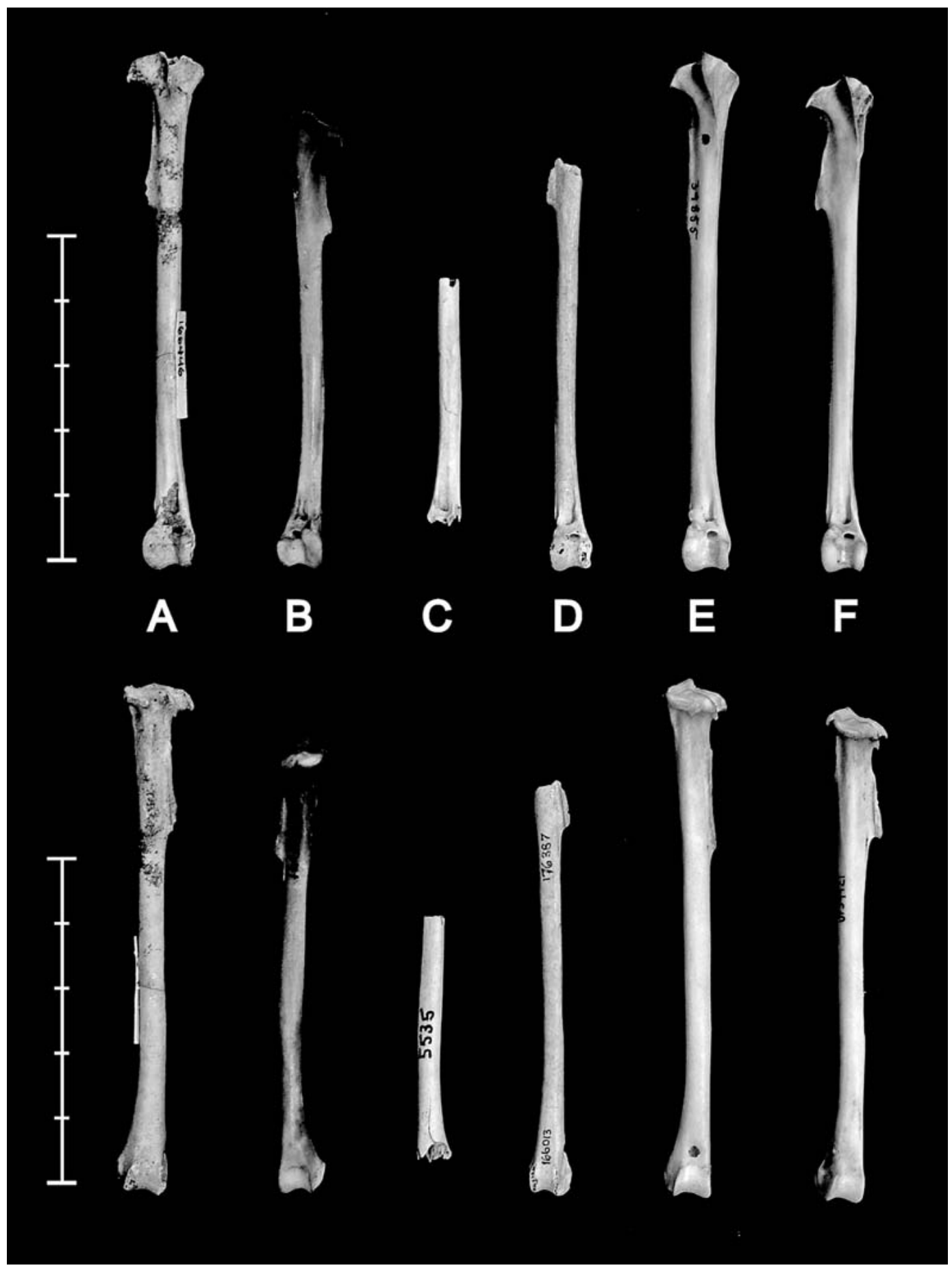

Figure 5. Tibiotarsi of $A$, $\dagger$ Gallirallus roletti (holotype, врвм 166446, Tahuata, Marquesas Islands); $B$, $\dagger$ G. storrsolsoni (врвм 166023, Huahine, Society Islands); $C, \dagger G$. epulare (врвм 181661, Nuku Hiva, Marquesas Islands); $D, \dagger G$. gracilitibia (holotype, врвм 166013/176387, Ua Huka, Marquesas Islands); E, G. philippensis (uf 39855, Tutuila, Samoa); and $F, G$. owstoni (Uf 39921, Guam, Mariana Islands) in dorsal (top) and ventral (bottom) aspects. Scale bars $=5 \mathrm{~cm}$. 


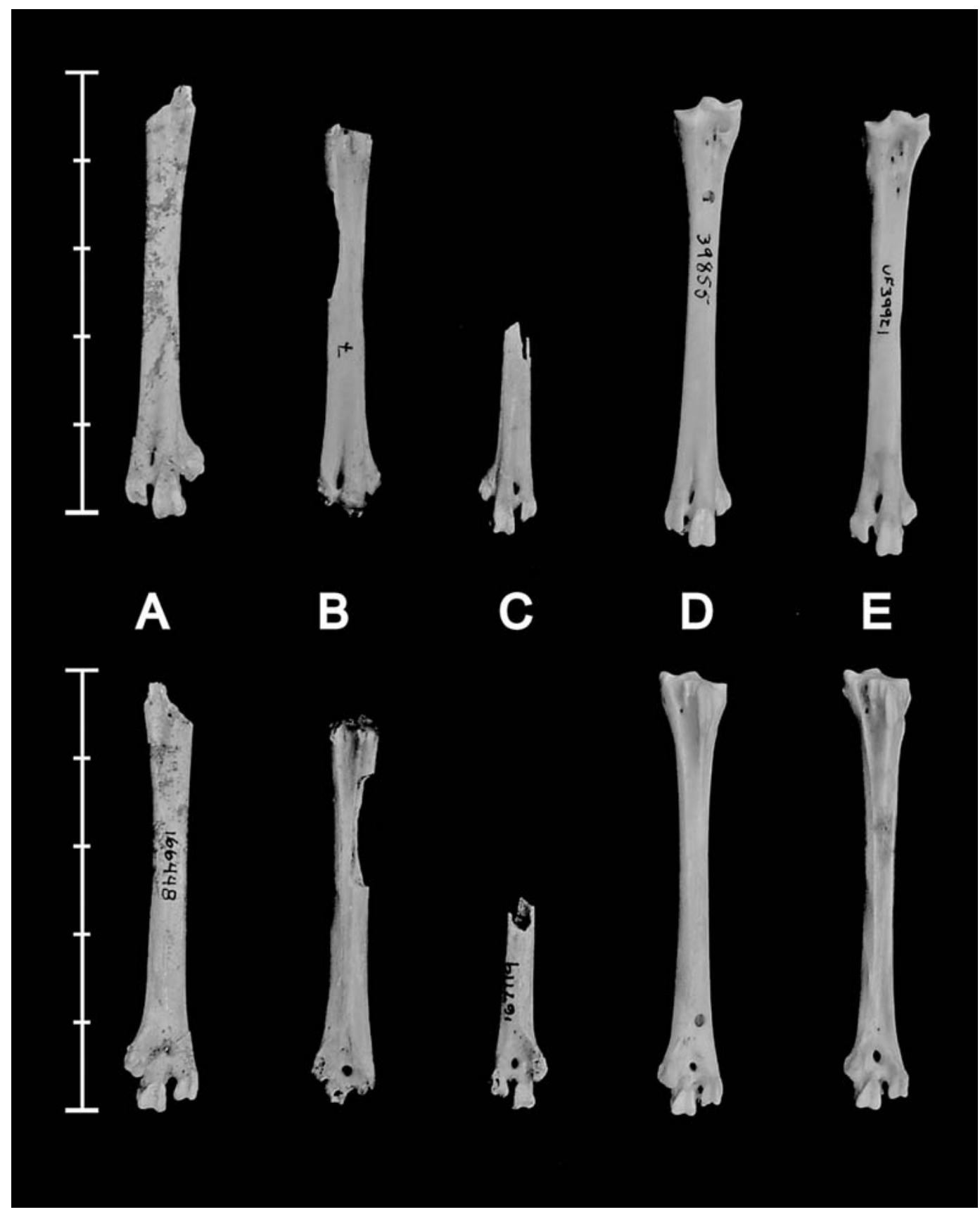

Figure 6. Tarsometatarsi of $A, \dagger$ Gallirallus roletti (holotype, врвм 166448, Tahuata, Marquesas Islands); $B, \dagger G$. storrsolsoni (DAPT 7, Huahine, Society Islands); $C, \dagger G$. epulare (врвм 167119, Nuku Hiva, Marquesas Islands); D, G. philippensis (uf 39855, Tutuila, Samoa); and E, G. owstoni (uf 39921, Guam, Mariana Islands) in acrotarsial (top) and plantar (bottom) aspects. Scale bars $=5 \mathrm{~cm}$. 


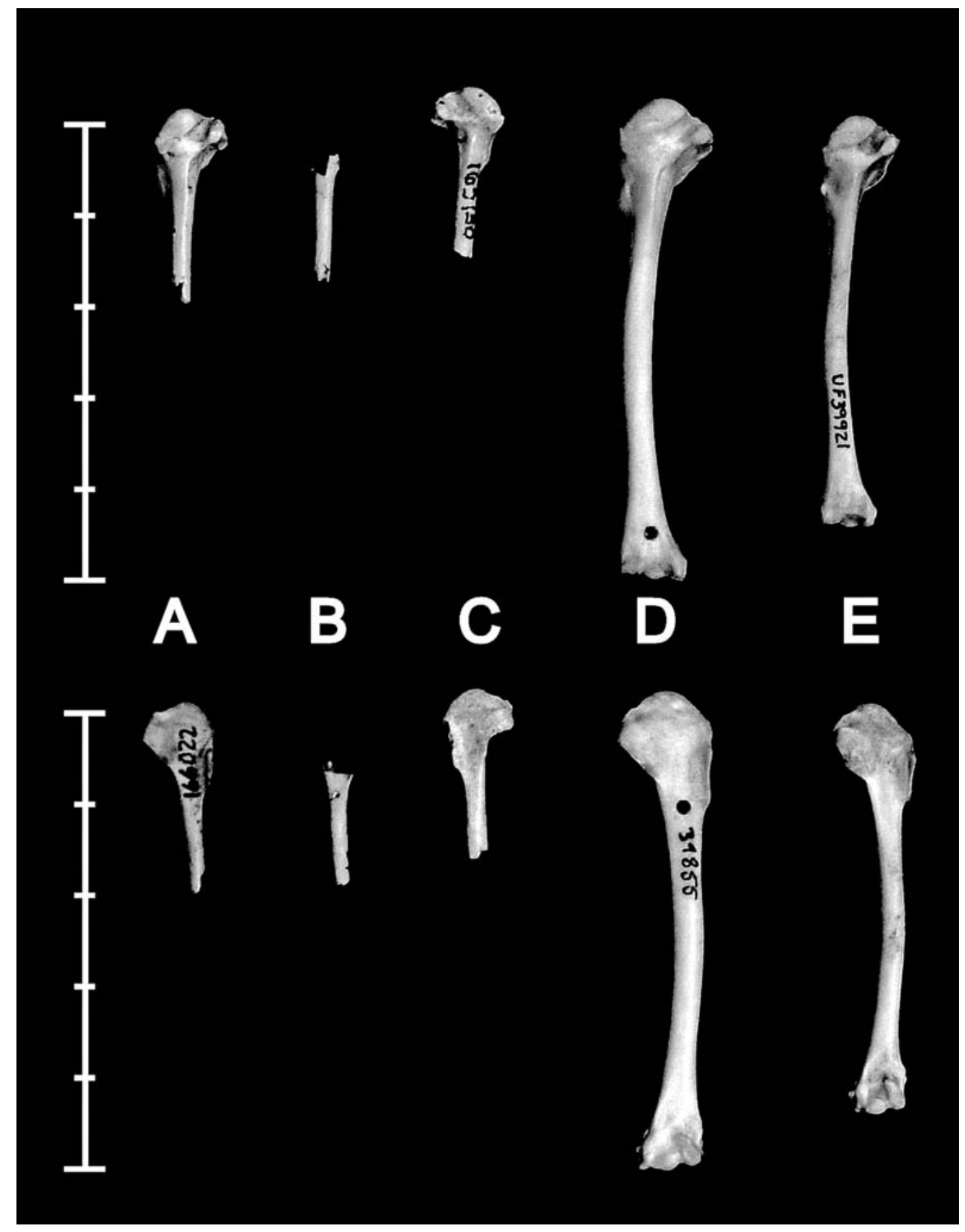

Figure 7. Humeri of $A, \dagger$ Gallirallus storrsolsoni (врвм 166022, Huahine, Society Islands); $B, \dagger$ G. epulare (врвм 181657, Nuku Hiva, Marquesas Islands); $C, \dagger G$. gracilitibia (врвм 163130, Ua Huka, Marquesas Islands); D, G. philippensis (uf 39855, Tutuila, Samoa); and E, G. owstoni (uf 39921, Guam, Mariana Islands) in ventral (top) and dorsal (bottom) aspects. Scale bars $=5 \mathrm{~cm}$. 
TABLE 1

Skeletal Measurements (in mm) in †Gallirallus roletti and Select Congeners, with Mean, Range, and Sample Size

\begin{tabular}{|c|c|c|c|c|c|c|c|c|}
\hline Parameter & $\begin{array}{l}\dagger G . \\
\text { roletti } \\
\mathrm{U}\end{array}$ & $\begin{array}{c}\dagger G . \\
\text { storrsolsoni } \\
\mathrm{U}\end{array}$ & $\begin{array}{c}G . \\
\text { owstoni } \\
M\end{array}$ & $\begin{array}{c}G . \\
\text { owstoni } \\
\mathrm{F}\end{array}$ & $\begin{array}{c}G . \\
\text { australis } \\
\mathrm{M}\end{array}$ & $\begin{array}{c}G . \\
\text { australis } \\
\mathrm{F}\end{array}$ & $\begin{array}{c}\dagger G . \\
\text { ripleyi } \\
\mathrm{U}\end{array}$ & $\begin{array}{c}\dagger G . \\
\text { vekamatolu } \\
\mathrm{U}\end{array}$ \\
\hline $\begin{array}{l}\text { Rostrum: height } \\
\text { at anterior } \\
\text { margin of } \\
\text { nares }\end{array}$ & $\begin{array}{l}5.2 \\
1\end{array}$ & $\begin{array}{l}3.7 \\
3.5-3.9 \\
2\end{array}$ & $\begin{array}{l}3.4 \\
3.2-3.8 \\
5\end{array}$ & $\begin{array}{l}3.3 \\
3.1-3.5 \\
4\end{array}$ & $\begin{array}{l}5.3 \\
5.1-5.4 \\
3\end{array}$ & $\begin{array}{l}4.7 \\
4.4-5.0 \\
4\end{array}$ & $\begin{array}{l}2.9 \\
1\end{array}$ & $\begin{array}{l}4.8 \\
1\end{array}$ \\
\hline $\begin{array}{l}\text { Rostrum: width } \\
\text { at anterior } \\
\text { margin of } \\
\text { nares }\end{array}$ & $\begin{array}{l}4.2 \\
1\end{array}$ & $\begin{array}{l}4.1 \\
4.0-4.2 \\
2\end{array}$ & $\begin{array}{l}4.4 \\
4.0-4.7 \\
5\end{array}$ & $\begin{array}{l}4.2 \\
3.8-4.5 \\
4\end{array}$ & $\begin{array}{l}5.5 \\
5.1-5.7 \\
3\end{array}$ & $\begin{array}{l}5.0 \\
4.5-5.5 \\
4\end{array}$ & $\begin{array}{l}2.8 \\
1\end{array}$ & $\begin{array}{l}5.9 \\
1\end{array}$ \\
\hline Femur: length & $\begin{array}{c}54.5 \\
1\end{array}$ & $\begin{array}{c}49.4 \\
1\end{array}$ & $\begin{array}{l}56.0 \\
54.0-59.2 \\
7\end{array}$ & $\begin{array}{l}52.4 \\
49.2-54.6 \\
11\end{array}$ & $\begin{array}{l}80.6 \\
78.7-82.3 \\
3\end{array}$ & $\begin{array}{l}69.9 \\
66.1-73.4 \\
4\end{array}$ & $\begin{array}{c}39.8 \\
1\end{array}$ & - \\
\hline $\begin{array}{l}\text { Femur: distal } \\
\text { width }\end{array}$ & $\begin{array}{l}9.8 \\
1\end{array}$ & $\begin{array}{l}9.2 \\
1\end{array}$ & $\begin{array}{l}9.5 \\
8.9-9.9 \\
7\end{array}$ & $\begin{array}{l}8.7 \\
8.4-9.0 \\
11\end{array}$ & $\begin{array}{l}16.5 \\
15.9-17.6 \\
3\end{array}$ & $\begin{array}{l}14.3 \\
13.2-15.0 \\
4\end{array}$ & $\begin{array}{l}6.7 \\
1\end{array}$ & $\begin{array}{l}10.9 \\
10.7-11.0 \\
2\end{array}$ \\
\hline $\begin{array}{l}\text { Femur: minimum } \\
\text { shaft width }\end{array}$ & $\begin{array}{l}4.0 \\
4.0 \\
2\end{array}$ & $\begin{array}{l}3.9 \\
1\end{array}$ & $\begin{array}{l}3.8 \\
3.3-4.0 \\
7\end{array}$ & $\begin{array}{l}3.6 \\
3.4-3.9 \\
11\end{array}$ & $\begin{array}{l}6.7 \\
6.5-6.9 \\
3\end{array}$ & $\begin{array}{l}5.6 \\
5.1-5.9 \\
4\end{array}$ & $\begin{array}{l}3.0 \\
1\end{array}$ & - \\
\hline $\begin{array}{l}\text { Tibiotarsus: } \\
\text { length }\end{array}$ & $\begin{array}{c}79.5 \\
1\end{array}$ & $\begin{array}{c}68.6 \\
1\end{array}$ & $\begin{array}{l}79.9 \\
76.1-84.6 \\
7\end{array}$ & $\begin{array}{l}75.5 \\
72.9-79.8 \\
11\end{array}$ & $\begin{array}{l}117.4 \\
116.6-118.7 \\
3\end{array}$ & $\begin{array}{l}100.0 \\
93.4-105.0 \\
4\end{array}$ & - & - \\
\hline $\begin{array}{l}\text { Tibiotarsus: } \\
\text { distal width }\end{array}$ & $\begin{array}{l}7.8 \\
7.7-7.9 \\
2\end{array}$ & $\begin{array}{l}6.9 \\
1\end{array}$ & $\begin{array}{l}7.4 \\
6.8-7.9 \\
7\end{array}$ & $\begin{array}{l}6.8 \\
6.5-7.1 \\
11\end{array}$ & $\begin{array}{l}12.5 \\
12.1-13.1 \\
3\end{array}$ & $\begin{array}{l}10.8 \\
10.4-11.2 \\
4\end{array}$ & $\begin{array}{l}6.0 \\
5.6-6.3 \\
3\end{array}$ & $\begin{array}{l}8.0 \\
7.9-8.0 \\
2\end{array}$ \\
\hline $\begin{array}{l}\text { Tarsometatarsus: } \\
\text { proximal width }\end{array}$ & $\begin{array}{l}7.9 \\
1\end{array}$ & $\begin{array}{l}7.4 \\
1\end{array}$ & $\begin{array}{l}7.6 \\
7.0-8.2 \\
7\end{array}$ & $\begin{array}{l}7.0 \\
6.7-7.3 \\
11\end{array}$ & $\begin{array}{l}12.6 \\
12.2-13.4 \\
3\end{array}$ & $\begin{array}{l}11.0 \\
10.7-11.7 \\
4\end{array}$ & $\begin{array}{l}5.7 \\
1\end{array}$ & $\begin{array}{l}8.3 \\
7.8-8.7 \\
2\end{array}$ \\
\hline $\begin{array}{l}\text { Tarsometatarsus: } \\
\text { distal width }\end{array}$ & $\begin{array}{l}8.7 \\
1\end{array}$ & - & $\begin{array}{l}8.0 \\
7.2-8.5 \\
7\end{array}$ & $\begin{array}{l}7.4 \\
7.2-7.8 \\
11\end{array}$ & $\begin{array}{l}14.0 \\
13.6-14.3 \\
3\end{array}$ & $\begin{array}{l}12.1 \\
11.2-13.0 \\
4\end{array}$ & $\begin{array}{l}6.4 \\
6.3-6.5 \\
3\end{array}$ & $\begin{array}{l}8.5 \\
8.0-9.0 \\
2\end{array}$ \\
\hline $\begin{array}{l}\text { Tarsometatarsus: } \\
\text { shaft width }\end{array}$ & $\begin{array}{l}3.5-3.9 \\
3.7 \\
2\end{array}$ & $\begin{array}{l}4.1 \\
1\end{array}$ & $\begin{array}{l}3.8 \\
3.5-4.0 \\
7\end{array}$ & $\begin{array}{l}3.4 \\
3.2-3.8 \\
11\end{array}$ & $\begin{array}{l}6.1 \\
5.6-6.4 \\
3\end{array}$ & $\begin{array}{l}5.4 \\
5.2-5.6 \\
4\end{array}$ & $\begin{array}{l}3.0 \\
2.8-3.1 \\
2\end{array}$ & $\begin{array}{l}4.3 \\
4.0-4.6 \\
2\end{array}$ \\
\hline $\begin{array}{l}\text { Tarsometatarsus: } \\
\text { shaft depth }\end{array}$ & $\begin{array}{l}2.7 \\
2.5-2.8 \\
2\end{array}$ & $\begin{array}{l}2.8 \\
1\end{array}$ & $\begin{array}{l}2.8 \\
2.5-3.0 \\
7\end{array}$ & $\begin{array}{l}2.5 \\
2.4-2.8 \\
11\end{array}$ & $\begin{array}{l}4.8 \\
4.5-5.0 \\
3\end{array}$ & $\begin{array}{l}4.1 \\
3.8-4.6 \\
4\end{array}$ & $\begin{array}{l}2.2 \\
2.1-2.3 \\
2\end{array}$ & $\begin{array}{l}3.2 \\
3.0-3.3 \\
2\end{array}$ \\
\hline
\end{tabular}

Note: Specimens of all available subspecies of G. australis and G. philippensis are combined, given that subspecific differences in size are much smaller than those between males and females. F, female; M, male; U, sex unknown; —, cannot be evaluated.

coid's small size (relative to leg elements) is due to flightlessness rather than to the age of the bird.

Steadman and Rolett (1996) referred 24 specimens to "Gallirallus new sp. (Tahuata Rail)," which is named herein as G. roletti, based on 22 specimens. The discrepancy is accounted for by an ungual phalanx of $\dagger$ Porphyrio paepae (врвм 166442) being mistakenly listed among the specimens of "Gallirallus new sp." in Steadman and Rolett
(1996) and by another pedal phalanx (врвм 166453) whose identity cannot now be determined.

$\dagger$ Gallirallus gracilitibia Kirchman \& Steadman, n. sp.

Figures $4 C, 5 D, 7 C$

HоLотуре. Right tibiotarsus lacking proximal end, врвм 166013, 176387 (Figure $5 D$; two pieces with different catalog numbers 
TABLE 1

(continued)

\begin{tabular}{|c|c|c|c|c|c|c|}
\hline $\begin{array}{c}G . \\
\text { philippensis } \\
M\end{array}$ & $\begin{array}{c}G . \\
\text { philippensis } \\
\mathrm{F}\end{array}$ & $\begin{array}{c}G . \\
\text { striatus } \\
\text { M }\end{array}$ & $\begin{array}{c}G . \\
\text { torquatus } \\
M\end{array}$ & $\begin{array}{c}G . \\
\text { torquatus } \\
\mathrm{F}\end{array}$ & $\begin{array}{c}G . \\
\text { woodfordi } \\
M\end{array}$ & $\begin{array}{c}G . \\
\text { woodfordi } \\
\mathrm{F}\end{array}$ \\
\hline 3.1 & 2.8 & 2.8 & 3.2 & - & 4.3 & 4.2 \\
\hline $2.8-3.4$ & $2.6-2.9$ & 1 & $3.1-3.3$ & & $4.2-4.6$ & $3.9-4.4$ \\
\hline 5 & 5 & & 2 & & 3 & 3 \\
\hline 3.7 & 3.2 & 3.1 & 3.4 & - & 4.7 & 4.6 \\
\hline $3.4-4.2$ & $3.1-3.4$ & 1 & $3.4-3.4$ & & $4.6-4.9$ & $4.5-4.8$ \\
\hline 5 & 5 & & 2 & & 3 & 3 \\
\hline 53.8 & 47.3 & 45.1 & 55.5 & 50.6 & 72.1 & 69.6 \\
\hline $51.3-54.9$ & $45.0-49.7$ & $42.2-46.9$ & $53.3-57.5$ & 1 & $72.0-72.3$ & $69.0-70.3$ \\
\hline 6 & 6 & 4 & 5 & & 3 & 3 \\
\hline 8.7 & 7.4 & 45.1 & 9.3 & 8.5 & 13.3 & 12.7 \\
\hline $8.4-9.1$ & $7.0-7.9$ & $42.2-46.9$ & $8.6-9.9$ & 1 & $13.2-13.4$ & $12.5-12.9$ \\
\hline 6 & 6 & 4 & 5 & & 3 & 3 \\
\hline 4.0 & 3.3 & 2.9 & 3.9 & 3.2 & 5.3 & 4.8 \\
\hline $3.7-4.2$ & $3.1-3.5$ & $2.8-3.1$ & $3.5-4.1$ & 1 & $5.2-5.3$ & $4.6-5.1$ \\
\hline 6 & 6 & 4 & 5 & & 3 & 3 \\
\hline 76.9 & 66.7 & 62.1 & 84.2 & 74.6 & 105.0 & 102.8 \\
\hline $71.7-80.3$ & $63.0-70.6$ & $58.2-64.3$ & $80.9-87.5$ & 1 & $100.6-108.8$ & $99.0-105.8$ \\
\hline 6 & 6 & 4 & 5 & & 3 & 3 \\
\hline 6.9 & 6.1 & 5.3 & 7.2 & 6.6 & 10.0 & 9.5 \\
\hline $6.5-7.2$ & $5.6-6.7$ & $5.1-5.6$ & $6.7-7.6$ & 1 & $9.9-10.1$ & $9.4-9.6$ \\
\hline 6 & 6 & 4 & 5 & & 3 & 3 \\
\hline 7.0 & 6.1 & 5.4 & 7.4 & 6.6 & 10.5 & 10.0 \\
\hline $6.7-7.2$ & $5.8-6.6$ & $5.3-5.5$ & $7.0-7.9$ & 1 & $10.2-10.7$ & $9.9-10.1$ \\
\hline 6 & 6 & 4 & 5 & & 3 & 3 \\
\hline 7.1 & 6.3 & 5.6 & 7.8 & 7.0 & 10.8 & 10.6 \\
\hline $6.7-7.5$ & $6.0-6.9$ & $5.4-5.7$ & $7.3-8.4$ & 1 & $10.7-11.0$ & $10.5-10.6$ \\
\hline 6 & 6 & 4 & 5 & & 3 & 3 \\
\hline 3.5 & 3.0 & 2.7 & 3.6 & 3.3 & 4.9 & 4.6 \\
\hline $3.1-3.6$ & $2.8-3.3$ & $2.5-2.9$ & $3.3-3.8$ & 1 & $4.8-5.0$ & $4.4-4.7$ \\
\hline 6 & 6 & 4 & 5 & & 3 & 3 \\
\hline 2.8 & 2.5 & 2.1 & 2.7 & 2.6 & 4.0 & 3.8 \\
\hline $2.6-3.0$ & $2.3-2.8$ & $2.0-2.2$ & $2.2-2.9$ & 1 & $3.9-4.2$ & $3.7-4.0$ \\
\hline 6 & 6 & 4 & 5 & & 3 & 3 \\
\hline
\end{tabular}

glued together). From the Hane Dune archaeological site, Ua Huka, Marquesas Islands. Collected by Y. H. Sinoto and colleagues on 11 October 1965.

PARATYPES. Right coracoid (humeral end), врвм 166015; left proximal humerus, вРвм 163130 (Figure 7C); right humerus shaft, вРвм 166014; right femur shaft, вРвм 176974 (Figure 4C); left distal tibiotarsi, врвм 163240, 166016, 169146, 170909; left tibiotarsus shaft, врвм 175384; right distal tibiotarsi, вРвм 166008, 166012, 169145 , 175145; right tibiotarsi shafts, врвм 171294, 175322, 175395, 176725, 176746, 176971, 176972. All are from the same locality as the holotype. A minimum of eight individuals is represented.

Diagnosis. A small to medium-sized (Table 2), flightless species of Gallirallus that differs from congeneric species as follows. Coracoid: sulcus musculo supracoracoidei relatively shallower and wider than in volant 
TABLE 2

Skeletal Measurements (in mm) of $\dagger$ Gallirallus gracilitibia and Selected Congeners with Mean, Range, and Sample Size

\begin{tabular}{|c|c|c|c|c|c|c|c|c|}
\hline Parameter & $\begin{array}{c}\dagger G . \\
\text { gracilitibia } \\
\mathrm{U}\end{array}$ & $\begin{array}{c}\dagger G . \\
\text { storrsolsoni } \\
\mathrm{U}\end{array}$ & $\begin{array}{c}G . \\
\text { philippensis } \\
M\end{array}$ & $\begin{array}{c}G . \\
\text { philippensis } \\
\mathrm{F}\end{array}$ & $\begin{array}{c}G . \\
\text { owstoni } \\
M\end{array}$ & $\begin{array}{c}G . \\
\text { owstoni } \\
\mathrm{F}\end{array}$ & $\begin{array}{c}G . \\
\text { torquatus } \\
M\end{array}$ & $\begin{array}{c}G . \\
\text { torquatus } \\
\text { F }\end{array}$ \\
\hline \multirow{3}{*}{$\begin{array}{l}\text { Humerus: shaft } \\
\text { width }\end{array}$} & 2.2 & 2.2 & 3.3 & 2.9 & 28 & 2.7 & & .9 \\
\hline & $2.1-2$ & 1 & $3.2-$ & 2.7 & & & $3.0-$ & 1 \\
\hline & 2 & & 5 & 6 & 7 & 11 & 5 & \\
\hline \multirow{3}{*}{$\begin{array}{l}\text { Tibiotarsus: shaft } \\
\text { width }\end{array}$} & 3.0 & 3.4 & 3.6 & 3.1 & 4.1 & 3.8 & 3.8 & 3.3 \\
\hline & 2. & $3.2-3$. & $3.3-4$ & $2.9-3$ & 3.8 & $3.4-4.1$ & $3.4-4.0$ & 1 \\
\hline & 8 & 3 & 6 & 6 & 7 & 11 & 5 & \\
\hline \multirow{2}{*}{$\begin{array}{l}\text { Tibiotarsus: length } \\
\text { to fibular } \text { crest }^{a}\end{array}$} & 54.5 & 50.2 & 52.8 & 47.1 & 57.8 & 54.4 & & 59.0 \\
\hline & 1 & $\begin{array}{l}50.2-50.2 \\
2\end{array}$ & $\begin{array}{l}51.9-55.3 \\
5\end{array}$ & $\begin{array}{l}43.3-51.6 \\
6\end{array}$ & $\begin{array}{l}55.2-61.2 \\
7\end{array}$ & $\begin{array}{l}52.9-55.9 \\
11\end{array}$ & $\begin{array}{c}59.3-61.5 \\
2\end{array}$ & 1 \\
\hline \multirow{3}{*}{$\begin{array}{l}\text { Tibiotarsus: distal } \\
\text { width }\end{array}$} & 6.4 & 6.9 & 6.9 & 6.1 & 7.4 & 6.8 & 7.2 & 6.6 \\
\hline & $6.3-6.5$ & 1 & $6.5-7.2$ & $5.6-6.7$ & $6.8-7.9$ & $6.5-7.1$ & $6.7-7.6$ & 1 \\
\hline & 4 & & 6 & 6 & 7 & 11 & 5 & \\
\hline
\end{tabular}

Note: F, female; M, male; U, sex unknown.

${ }^{a}$ Distal end of bone to distal edge of fibular crest.

species. Humerus (Figure 7C): crista bicipitalis small; corpus humeri thin and curved; distal junction of crista pectoralis and corpus humeri abrupt, rather than gradually sloping; sulcus ligamentum transversus deep. Femur (Figure 4C): corpus femoris gracile. Tibiotarsus (Figure 5D): corpus tibiotarsus slender relative to its length; depressio epicondylaris medialis deep.

ETYMOLOGY. From the Latin words gracilis (slender, slim, thin) and tibia (the shinbone, tibia); see Brown (1956:469, 791). The name gracilitibia is a feminine noun in apposition to Gallirallus. It refers to the distinctively slender tibiotarsus in this species, especially compared with that of $\dagger G$. roletti.

REMARKs. †Gallirallus gracilitibia is a small to medium-sized species (Table 2), exceeded in stoutness of skeletal elements by all congeners but resembling the medium-sized $G$. owstoni, G. philippensis, and G. torquatus in measurements along the long axes of leg elements. Based on length-to-width ratios of tibiotarsi, $\dagger G$. gracilitibia has the thinnest shaft relative to length of any species of Gallirallus, whether flightless or volant (Table 2). Described features of the coracoid and humerus of $\dagger G$. gracilitibia, as well as their size relative to hind limb elements (see Livezey 2003,
Kirchman and Steadman 2005, 2006), indicate that this species was flightless.

$\dagger$ Gallirallus epulare Kirchman \& Steadman, n. sp.

Figures $4 D, 5 C, 6 C, 7 B, 8 B$

holotype. Nearly complete left ulna, врвм 181658 (Figure 8B). From the $\mathrm{Ha}^{\prime}$ atuatua archaeological site, Nuku Hiva, Marquesas Islands. Collected by B. V. Rolett, E. Conte, and colleagues in 1994-1995.

PaRATYPES. Left humerus shaft, врвм 181657 (Figure 7B); left femur shaft, врвм 181659 (Figure 4D); left tibiotarsi shafts, врвм 181660, 181661 (Figure 5C), 181662; right tibiotarsus shaft, врвм 167191; left distal tarsometatarsus, вРвм 167119 (Figure 6C). All are from the same locality as the holotype.

DiAgnosis. A small species of Gallirallus distinguished from congeneric species as follows. Humerus and ulna very small and slender relative to leg elements, especially compared with those of $\dagger G$. gracilitibia from nearby Ua Huka. Ulna: straighter than in volant species. Tarsometatarsus: facies dorsalis of corpus tarsometatarsi highly convex proximal to trochlea metatarsi III; foramen vasculare distale relatively large and round. 


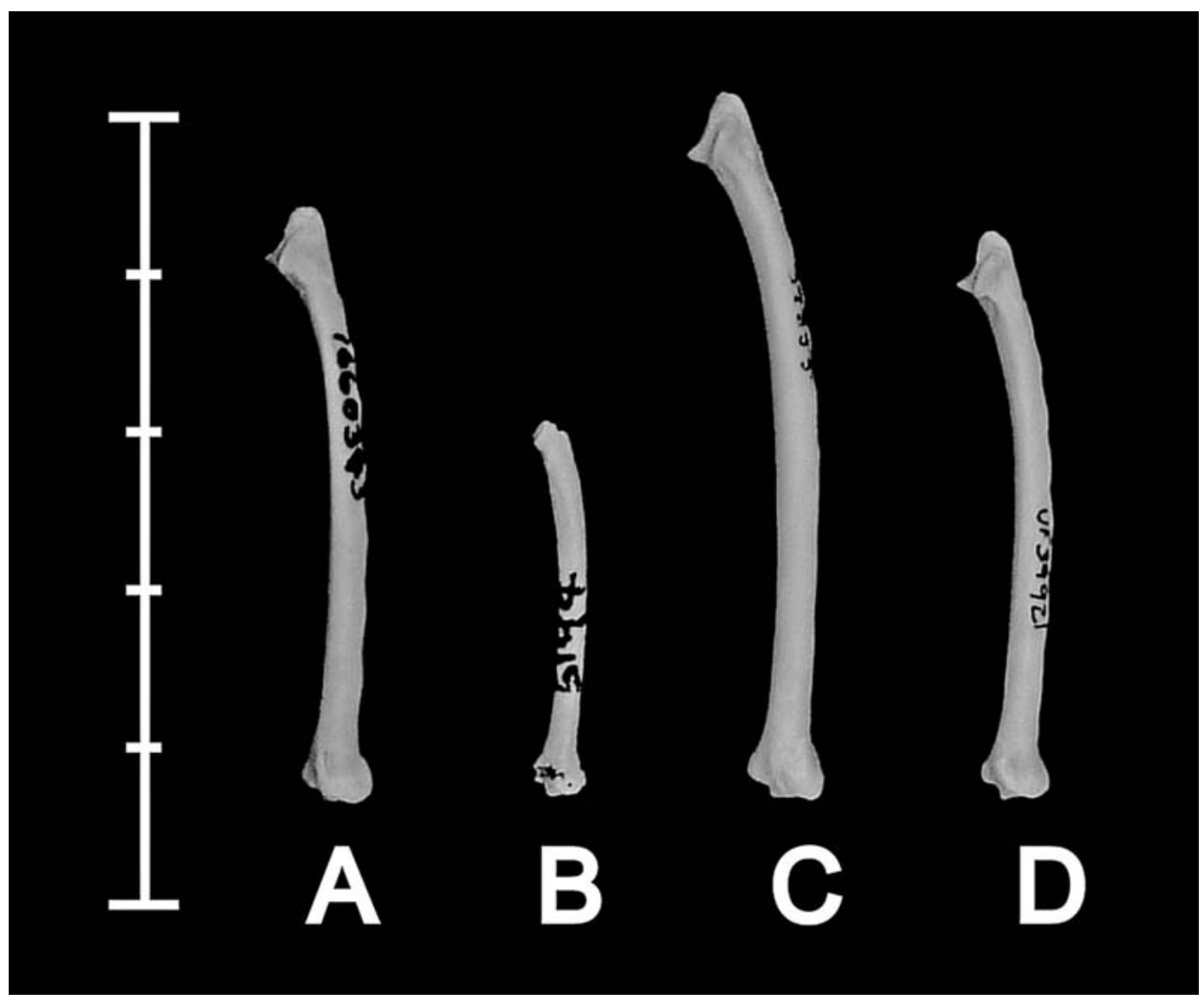

Figure 8. Ulnae of $A$, †Gallirallus storrsolsoni (врвм 166033, Huahine, Society Islands); $B, \dagger G$. epulare (holotype, врвм 181658, Nuku Hiva, Marquesas Islands); C, G. philippensis (uf 39855, Tutuila, Samoa); and D, G. owstoni (uf 39921, Guam, Mariana Islands) in dorsal aspect. Scale bar $=5 \mathrm{~cm}$.

etymology. From the Latin epularis (pertaining to a banquet, belonging to a banquet [Brown 1956:292]). The name epulare is an adjective that modifies the masculine $\mathrm{Gal}$ lirallus. It refers to the archaeological context in which the specimens of $\dagger G$. epulare were found, namely that of a kitchen midden dominated by the bony and shelly remains of foods eaten by prehistoric Polynesians.

REMARKs. The humerus, femur, and tibiotarsi lack both the proximal and distal ends and therefore also lack diagnostic features. The most striking feature of $\dagger$ Gallirallus epulare is its tiny wing elements relative to its leg elements; the leg elements closely resemble those from a small female
G. owstoni, but the humerus and ulna are much smaller than those of G. owstoni and indeed more closely resemble those of $\dagger G$. ripleyi and $\dagger G$. wakensis.

\section{cf. $\dagger$ Gallirallus sp.}

Figures $4 B, 9 A$

material. Complete left scapula, врвм 165655 (Figure 9A); shaft of right femur, врвм 168539 (Figure 4B). From the Hanatekua Rockshelter archaeological site, Hiva Oa, Marquesas Islands. Collected by Y. H. Sinoto and colleagues in 1965-1966.

REMARKs. These two bones represent a large, probably flightless species of Gallirallus. 
TABLE 3

Skeletal Measurements (in mm) of $\dagger$ Gallirallus epulare and Selected Congeners with Mean, Range, and Sample Size

\begin{tabular}{|c|c|c|c|c|c|c|c|c|}
\hline Parameter & $\begin{array}{c}\dagger G . \\
\text { epulare } \\
\mathrm{U}\end{array}$ & $\begin{array}{c}\dagger G . \\
\text { storrsolsoni } \\
\mathrm{U}\end{array}$ & $\begin{array}{c}G . \\
\text { philippensis } \\
M\end{array}$ & $\begin{array}{c}G . \\
\text { philippensis } \\
\mathrm{F}\end{array}$ & $\begin{array}{c}G . \\
\text { owstoni } \\
\text { M }\end{array}$ & $\begin{array}{c}G . \\
\text { owstoni } \\
\mathrm{F}\end{array}$ & $\begin{array}{c}G . \\
\text { torquatus } \\
M\end{array}$ & $\begin{array}{c}G . \\
\text { torquatus } \\
\mathrm{F}\end{array}$ \\
\hline \multirow[t]{2}{*}{ Ulna: length } & $25.5^{*}$ & 37.5 & 43.9 & 39.2 & 39.1 & 36.6 & 44.0 & 40.0 \\
\hline & 1 & 1 & $\begin{array}{l}41.4-44.8 \\
6\end{array}$ & $\begin{array}{l}35.2-43.6 \\
6\end{array}$ & $\begin{array}{l}36.8-41.5 \\
7\end{array}$ & $\begin{array}{l}34.8-38.7 \\
11\end{array}$ & $\begin{array}{l}43.0-45.3 \\
5\end{array}$ & 1 \\
\hline \multirow[t]{2}{*}{ Ulna: shaft width } & 2.0 & 2.7 & 3.0 & 2.7 & 3.0 & 2.7 & 3.0 & 2.8 \\
\hline & 1 & 1 & $\begin{array}{l}2.8-3.1 \\
6\end{array}$ & $\begin{array}{l}2.6-2.8 \\
6\end{array}$ & $\begin{array}{l}2.8-3.2 \\
7\end{array}$ & $\begin{array}{l}2.6-3.0 \\
11\end{array}$ & $\begin{array}{l}2.8-3.1 \\
5\end{array}$ & 1 \\
\hline \multirow{2}{*}{$\begin{array}{l}\text { Tibiotarsus: shaft } \\
\text { width }\end{array}$} & 3.4 & 3.4 & 3.6 & 3.1 & 4.1 & 3.8 & 3.8 & 3.3 \\
\hline & 1 & $\begin{array}{l}3.2-3.7 \\
3\end{array}$ & $\begin{array}{l}3.3-4.0 \\
6\end{array}$ & $\begin{array}{l}2.9-3.3 \\
6\end{array}$ & $\begin{array}{l}3.8-4.5 \\
7\end{array}$ & $\begin{array}{l}3.4-4.1 \\
11\end{array}$ & $\begin{array}{l}3.4-4.0 \\
5\end{array}$ & 1 \\
\hline \multirow{2}{*}{$\begin{array}{l}\text { Tarsometatarsus: } \\
\text { shaft width }\end{array}$} & 3.5 & 4.1 & 3.5 & 3.0 & 3.8 & 3.4 & 3.6 & 3.3 \\
\hline & 1 & 1 & $\begin{array}{l}3.1-3.6 \\
6\end{array}$ & $\begin{array}{l}2.8-3.3 \\
6\end{array}$ & $\begin{array}{l}3.5-4.0 \\
7\end{array}$ & $\begin{array}{l}3.2-3.8 \\
11\end{array}$ & $\begin{array}{l}3.3-3.8 \\
5\end{array}$ & 1 \\
\hline \multirow{2}{*}{$\begin{array}{l}\text { Tarsometatarsus: } \\
\text { shaft depth }\end{array}$} & 2.4 & 2.8 & 2.8 & 2.5 & 2.8 & 2.5 & 2.7 & 2.6 \\
\hline & 1 & 1 & $\begin{array}{l}2.6-3.0 \\
6\end{array}$ & $\begin{array}{l}2.3-2.8 \\
6\end{array}$ & $\begin{array}{l}2.5-3.0 \\
7\end{array}$ & $\begin{array}{l}2.4-2.8 \\
11\end{array}$ & $\begin{array}{l}2.2-2.9 \\
5\end{array}$ & 1 \\
\hline $\begin{array}{l}\text { Tarsometatarsus: } \\
\text { distal width }\end{array}$ & $\begin{array}{l}6.5 \\
1\end{array}$ & - & $\begin{array}{l}7.1 \\
6.7-7.5 \\
6\end{array}$ & $\begin{array}{l}6.3 \\
6.0-6.9 \\
6\end{array}$ & $\begin{array}{l}8.0 \\
7.2-8.5 \\
7\end{array}$ & $\begin{array}{l}7.4 \\
7.2-7.6 \\
11\end{array}$ & $\begin{array}{l}7.8 \\
7.3-8.4 \\
5\end{array}$ & $\begin{array}{l}7.0 \\
1\end{array}$ \\
\hline
\end{tabular}

Note: F, female; M, male; U, sex unknown; - , cannot be evaluated.

${ }^{*}$ Estimated.

We regard these bones as an inadequate basis for describing a new species but note that the scapula differs from that in all congeneric species in having a relatively thick area between the facies articularis humeralis and facies articularis clavicularis, in proximal aspect, and in having a distinctive flange on ventral surface of corpus scapulae near extremitas caudalis. The femur lacks both the proximal and distal ends but is remarkable in having a relatively wide divergence of the two linea intermuscularis caudalis.

The scapulae of the three named Marquesan species of Gallirallus and of †Porphyrio paepae (formerly of Tahuata and Hiva Oa) are unknown, thus precluding direct comparison with врвм 165655. The femoral shaft from Hiva Oa (врвм 168539 [Figure 4B]) is too large to belong to $\dagger P$. paepae and also is larger than that of $\dagger G$. roletti, suggesting that the Gallirallus species from Hiva $\mathrm{Oa}$ was quite large and that Gallirallus from the southern islands were larger than those of the two northern islands.

\section{DISCUSSION}

The rail bones from archaeological sites on three Marquesan islands (Tahuata, Nuku Hiva, and Ua Huka) represent three new flightless species of Gallirallus, one endemic to each island. A probable fourth species, from Hiva $\mathrm{Oa}$, requires more material before a description is possible. No species of Gallirallus exist anywhere today in East Polynesia, where all known species are flightless and extinct. They are $\dagger G$. ripleyi from Mangaia, Cook Islands; $\dagger G$. storrsolsoni from Huahine, Society Islands (both known only from bones from archaeological sites [Steadman 1987, Kirchman and Steadman 2006]); and $\dagger G$. pacificus from Tahiti, Society Islands, which survived to the eighteenth century (Ripley 1977, Taylor 1998).

The species from the Marquesas Islands represent the northwestern limit of Gallirallus. The very rich prehistoric record of birds $(15,000+$ bones from 12 archaeological sites) from Henderson Island (Pitcairn Group) has 


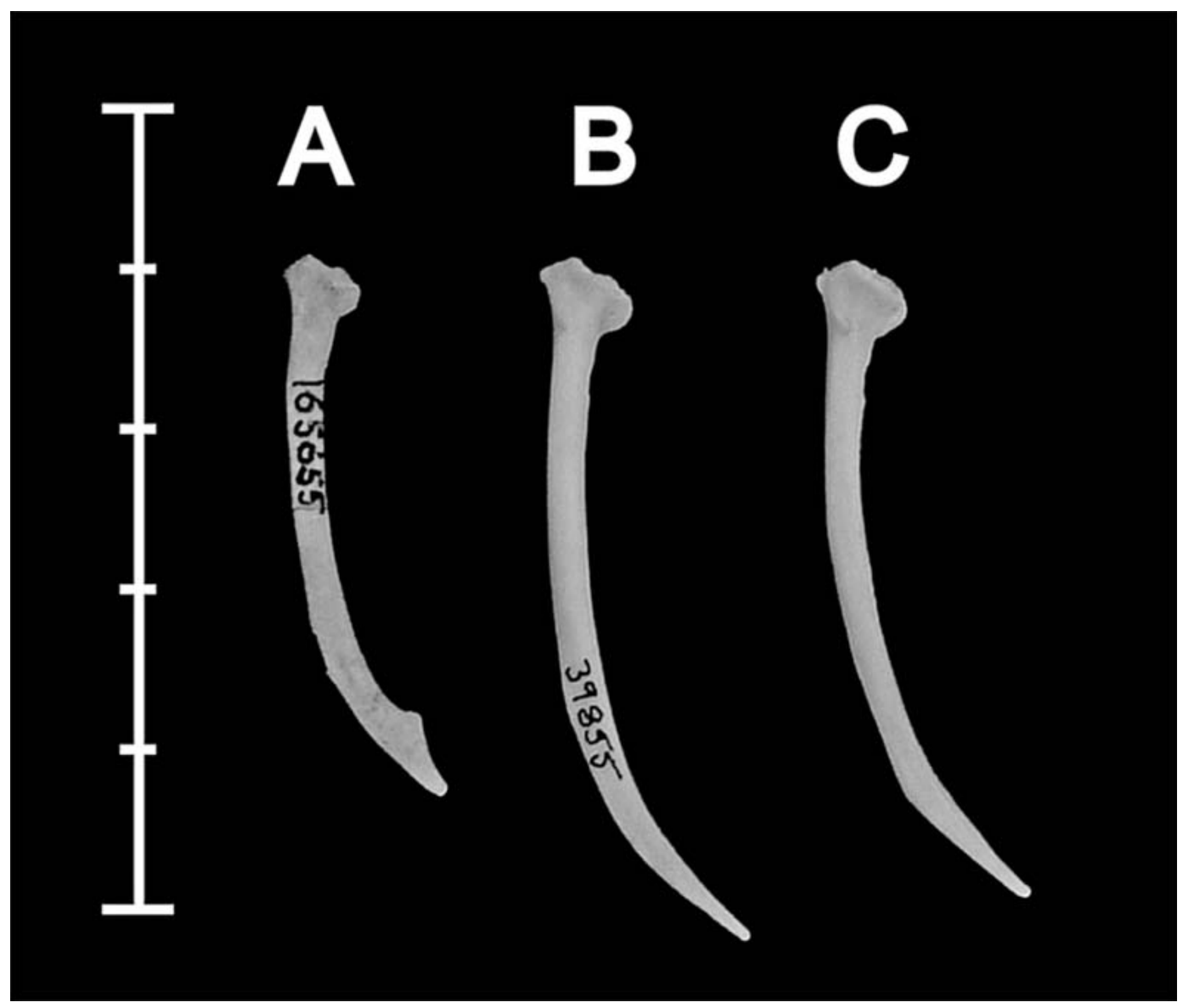

Figure 9. Scapulae of $A$, cf. $\dagger$ Gallirallus sp. (врвм 165655, Hiva Oa, Marquesas Islands); $B$, G. philippensis (uf 39855, Tutuila, Samoa); and C, G. owstoni (uf 39921, Guam, Mariana Islands) in dorsal aspect. Scale bar $=5 \mathrm{~cm}$.

not yielded evidence of Gallirallus (Steadman and Olson 1985, Wragg 1995). Between the Cook Islands (where $\dagger G$. ripleyi lived) and Henderson Island lie the Austral (Tubuai) Islands, where the single prehistoric land bird bone known represents an undescribed, extinct species of Ptilinopus (Columbidae) (Steadman 2006). Likewise, the well-studied fossils from dunes, lava tubes, and lakes in the Hawaiian Islands, which have yielded 7-10 flightless species of Porzana rails, lack evidence of Gallirallus (Olson and James 1991).

Aside from the species of Gallirallus, extinct species of Marquesan land birds known only from prehistoric bones are the swamphen †Porphyrio paepae, an undescribed species of sandpiper (Prosobonia), two doves $(\dagger$ Gallicolumba nui and $\dagger$ Macropygia beana), and two species of parrots ( $\dagger$ Vini vidivici and $\dagger V$. sinotoi) (Steadman and Zarriello 1987, Steadman 1988, 1992, 2006). The extinction of flightless rails in the Marquesas probably took place in prehistoric times. On Tahuata, all but 1 of the 22 specimens of $\dagger$ Gallirallus roletti were excavated from Levels G, GH, or $\mathrm{H}$ (Phases I and II) at Hanamiai, which represent the early occupation of the site at ca. 1,000-700 yr B.P. (Rolett 1998). A single bone of $\dagger G$. roletti is from Phase III (Level 
F), which dates to $700-550 \mathrm{yr}$ B.P. This may be the approximate time of extinction for $\dagger G$. roletti, bones of which were not recovered in the younger Levels A-D (Phases IV, V). Similarly on Ua Huka, all of the bones of $\dagger G$. gracilitibia were from strata dated to $>800$ yr B.P. (Steadman 1991).

\section{ACKNOWLEDGMENTS}

For access to modern and fossil specimens, we thank Paul Sweet (AмNн), James Dean and Storrs Olson (UsNM), Carla Kishinami (врвм), Alan Tennyson (NMNZ), Barry Rolett (University of Hawai'i), Tom Webber and Andrew Kratter (Uf), Janet Hinshaw (UMmz), Sievert Rohwer and Chris Wood (uwBm), and Kristof Zyskowski (YPM). For helpful comments on the manuscript, we thank Richard Holdaway and an anonymous reviewer.

\section{Literature Cited}

Baumel, J. L., and L. M. Witmer. 1993. Osteologia. Pages 45-132 in J. J. Baumel, A. S. King, J. E. Breazile, H. E. Evans, and J. C. Vanden Berge, eds. Handbook of avian anatomy: Nomina anatomica avium. 2nd ed. Nuttall Ornithological Club, Cambridge, Massachusetts.

Bellwood, P. 1972. Settlement pattern survey, Hanatekua Valley, Hiva Oa, Marquesas Islands. Pac. Anthropol. Rec. 17.

Brown, R. W. 1956. Composition of scientific words. Smithsonian Institution Press, Washington, D.C.

James, H. F., and S. L. Olson. 1991. Description of thirty-two new species of birds from the Hawaiian Islands: Part II. Passeriformes. Ornithol. Monogr. 46.

Kirchman, J. J., and D. W. Steadman. 2005. Rails (Aves: Rallidae: Gallirallus) from prehistoric sites in the Kingdom of Tonga, including description of a new species. Proc. Biol. Soc. Wash. 118:465-477.

. 2006. New species of rails (Aves: Rallidae) from an archaeological site on Huahine, Society Islands. Pac. Sci. 60:281297.

Livezey, B. C. 1998. A phylogenetic analysis of the Gruiformes (Aves) based on morphological characters, with an emphasis on the rails (Rallidae). Philos. Trans. R. Soc. Lond. B Biol. Sci. 353:2077-2151. . 2003. Evolution of flightlessness in rails (Gruiformes: Rallidae): Phylogenetic, ecomorphological, and ontogenetic perspectives. Ornithol. Monogr. 53.

Olson, S. L. 1973. A classification of the Rallidae. Wilson Bull. 85:381-416.

Olson, S. L., and H. F. James. 1991. Description of thirty-two new species of birds from the Hawaiian Islands: Part I. NonPasseriformes. Ornithol. Monogr. 45.

Pimm, S. L., M. P. Moulton, and L. J. Justice. 1994. Bird extinctions in the central $\mathrm{Pa}-$ cific. Philos. Trans. R. Soc. Lond. B Biol. Sci. 344:27-33.

Ripley, S. D. 1977. Rails of the world. Godine, Boston, Massachusetts.

Rolett, B. V. 1998. Hanamiai: Prehistory, colonization, and cultural change in the Marquesas Islands (East Polynesia). Yale Univ. Publ. Anthropol. 81.

Sinoto, Y. H. 1966. A tentative prehistoric cultural sequence in the northern Marquesas Islands, French Polynesia. J. Polynesian Soc. 75:287-303.

- 1970. An archaeologically based assessment of the Marquesas Islands as a dispersal center in East Polynesia. Pac. Anthropol. Rec. 11:105-132.

1979. The Marquesas. Pages 110 134 in J. Jennings, ed. The prehistory of Polynesia. Harvard University Press, Cambridge, Massachusetts.

Steadman, D. W. 1987. Two new species of rails (Aves: Rallidae) from Mangaia, southern Cook Islands. Pac. Sci. 40:38-54.

- 1988. A new species of Porphyrio (Aves: Rallidae) from archaeological sites in the Marquesas Islands. Proc. Biol. Soc. Wash. 101:162-170.

- 1989. Extinction of birds in eastern Polynesia: A review of the record, and comparisons with other Pacific island groups. J. Archaeol. Sci. 16:177-205.

- 1991. Extinction of species: Past, present, and future. Pages 156-170 in R. L. Wyman, ed. Global climate change and life on Earth. Routledge, Chapman and Hall, New York. 
1992. New species of Gallicolumba and Macropygia (Aves: Columbidae) from archaeological sites in Polynesia. Los Angel. Cty. Mus. Nat. Hist. Sci. Ser. 36:329-348.

- 1995. Prehistoric extinctions of Pacific island birds: Biodiversity meets zooarchaeology. Science (Washington, D.C.) 267:1123-1131.

- 2006. Extinction and biogeography of tropical Pacific birds. University of Chicago Press, Chicago, Illinois.

Steadman, D. W., and S. L. Olson. 1985. Bird remains from an archaeological site on Henderson Island, South Pacific: Mancaused extinctions on an "uninhabited" island. Proc. Natl. Acad. Sci. U.S.A. 82:6191-6195.

Steadman, D. W., G. K. Pregill, and D. V. Burley. 2002. Rapid prehistoric extinction of iguanas and birds in Polynesia. Proc. Natl. Acad. Sci. U.S.A. 99:3673-3677.

Steadman, D. W., and B. Rolett. 1996. A chronostratigraphic analysis of landbird extinction on Tahuata, Marquesas Islands. J. Archaeol. Sci. 23:81-94.

Steadman, D. W., T. H. Worthy, A. J. An- derson, and R. Walter. 2000. New species and records of birds from prehistoric sites on Niue, Southwest Pacific. Wilson Bull. 112:165-186.

Steadman, D. W., and M. C. Zarriello. 1987. Two new species of parrots (Aves: Psittacidae) from archaeological sites in the Marquesas Islands. Proc. Biol. Soc. Wash. 100:518-528.

Suggs, R. 1961. Archaeology of Nuku Hiva, Marquesas Islands, French Polynesia. Anthropol. Pap. Am. Mus. Nat. Hist. 49.

Taylor, P. B. 1998. Rails: A guide to the rails, crakes, gallinules and coots of the world. Yale University Press, New Haven, Connecticut.

Worthy, T. H. 2004. The fossil rails (Aves: Rallidae) of Fiji with descriptions of a new genus and species. J. R. Soc. N. Z. 34:295314.

Worthy, T. H., and R. N. Holdaway. 2002. The lost world of the moa. Indiana University Press, Bloomington.

Wragg, G. M. 1995. The fossil birds of Henderson Island, Pitcairn Group: Natural turnover and human impact, a synopsis. Biol. J. Linn. Soc. 56:405-414. 
